\title{
Heart Rate and Heart Rate Variability as Indicators of Stress in Emergency Medicine Residents during Simulation
}

\author{
Chase Hinman \& Shane Jenks, MD, MEd
}

\section{POSTER PRESENTATION ABSTRACT | ORIGINAL RESEARCH CATEGORY}

Introduction: This project was completed through collaboration between a Rice University Collegiate EMS research course and a Baylor College of Medicine EM Physician. When confronted with a stressful event, the human body responds by activating the sympathetic nervous system pathway. Studies have shown a relationship between stress-induced heart rate increases and cognitive performance. Certain cognitive tasks, such as memory recall and information processing, in many cases are negatively affected by such stress responses. Significant stress response has been shown in emergency medicine residents during regular shifts.

Methods: We measured stress-induced heart rate and heart rate variability of Baylor College of Medicine emergency medicine residents during a simulated case, specifically, a pulseless electrical activity cardiac arrest scenario. In addition to these vitals, and a few others, we also tested the resident's cognitive performance before and during the simulation. To measure physiological signs of stress we had the participants wear an Empatica E4 wristband. For cognitive performance level, the participants completed a trailmaking test.

Results: Our data showed insignificant signs of stress levels during the case, but the cognitive performance level was significantly better during the case as compared to before.

Discussion/Conclusions: The data is consistent with the conclusion that simulation cases do not create stress levels seen during shift, but they may mimic cognitive improvement. This conclusion has interesting implications for collegiate EMS, in reference to training exercises and testing using scenarios. An important future study could perform similar tests but on EMTs.

Author Affiliations: From Rice University \& Baylor College of Medicine - both in Houston, TX, USA (C.H. \& S.J.).

Address for Correspondence: Chase Hinman | Email: cmh13@rice.edu

Conflicts of Interest/Funding Sources: By the JCEMS Submission Declaration Form, all authors are required to disclose all potential conflicts of interest and funding sources. The authors declared that they have no conflicts of interest. The authors declared that they did not receive funding to conduct the program or research associated with this work.

Ethical Compliance: The authors attest that the research associated with this abstract was conducted in accordance with the JCEMS Ethics Guidelines.

Submission History: Received February 6, 2020; accepted for presentation and publication February 18, 2020.

Poster Presentation: This abstract was presented as a poster at the Academic Poster Session of the 27th Annual Conference of the National Collegiate Emergency
Medical Services Foundation; February 29, 2020; Boston, MA, USA.

Published Online: December 31, 2020

Published in Print: December 31, 2020 (Volume 3: Issue 2)

Reviewer Information: In accordance with JCEMS editorial policy, poster presentation abstracts undergo double-blind peer-review by at least two reviewers (JCEMS Editorial Board members and/or independent reviewers) prior to acceptance for presentation and publication. JCEMS thanks the anonymous reviewers who contributed to the review of this work.

Copyright: $\odot 2020$ Hinman. This is an open access article distributed under the terms of the Creative Commons Attribution 4.0 International (CC BY 4.0) License, which permits unrestricted use, distribution, and reproduction in any medium, provided the original author and source are credited. The full license is available at: https://creativecommons.org/licenses/by/4.0/

Electronic Link: https://doi.org/10.30542/JCEMS.2020.03.S1.05 